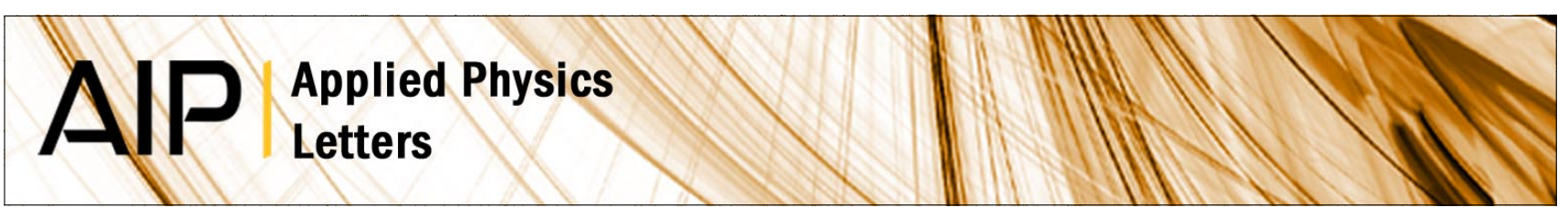

\title{
Optical detection of plasmonic and interband excitations in 1-nm- wide indium atomic wires
}

H. V. Chung, C. J. Kubber, G. Han, S. Rigamonti, D. Sanchez-Portal et al.

Citation: Appl. Phys. Lett. 96, 243101 (2010); doi: 10.1063/1.3451461

View online: http://dx.doi.org/10.1063/1.3451461

View Table of Contents: http://apl.aip.org/resource/1/APPLAB/v96/i24

Published by the American Institute of Physics.

\section{Related Articles}

On the origin of enhanced photoconduction and photoluminescence from Au and Ti nanoparticles decorated aligned $\mathrm{ZnO}$ nanowire heterostructures

J. Appl. Phys. 110, 124317 (2011)

Raman scattering from Ge-Si core-shell nanowires: Validity of analytical strain models

J. Appl. Phys. 110, 124305 (2011)

Surface effects in a semiconductor photonic nanowire and spectral stability of an embedded single quantum dot Appl. Phys. Lett. 99, 233106 (2011)

Strong modification of the reflection from birefringent layers of semiconductor nanowires by nanoshells Appl. Phys. Lett. 99, 201108 (2011)

Optical and electrical properties of gold nanowires synthesized by electrochemical deposition

J. Appl. Phys. 110, 094301 (2011)

\section{Additional information on Appl. Phys. Lett.}

Journal Homepage: http://apl.aip.org/

Journal Information: http://apl.aip.org/about/about_the_journal

Top downloads: http://apl.aip.org/features/most_downloaded

Information for Authors: http://apl.aip.org/authors

\section{ADVERTISEMENT}

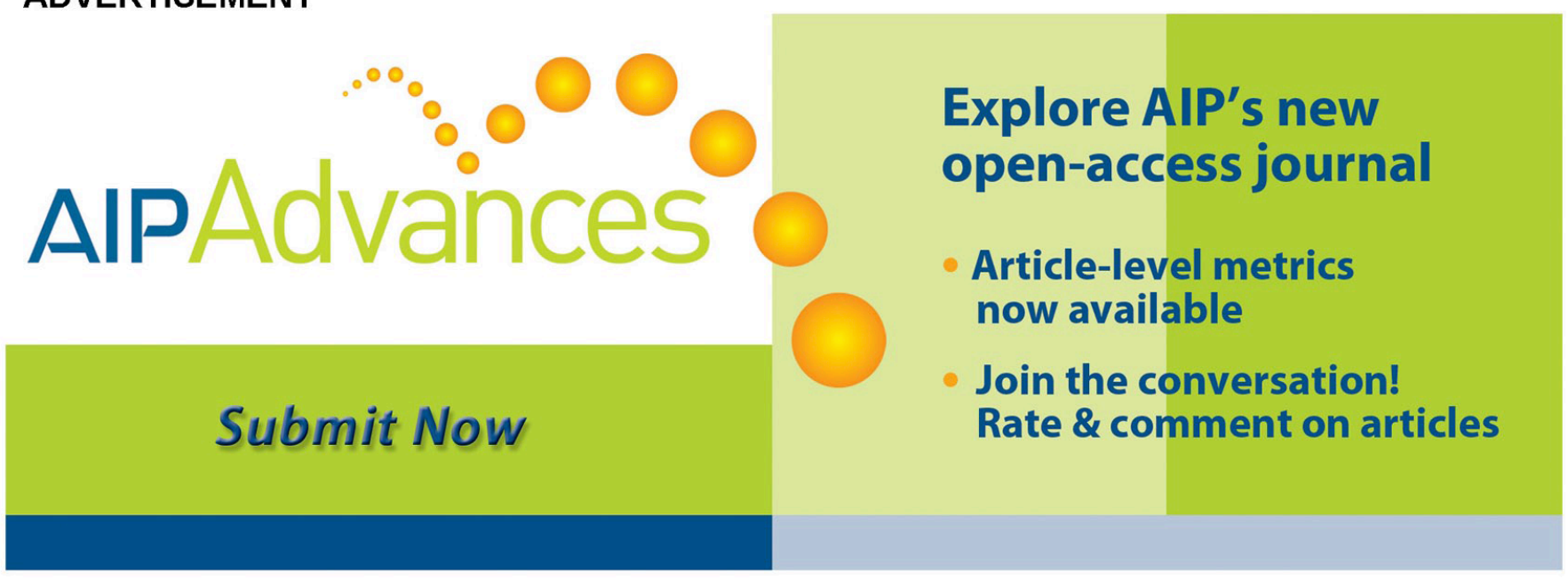




\title{
Optical detection of plasmonic and interband excitations in 1-nm-wide indium atomic wires
}

\author{
H. V. Chung, ${ }^{1}$ C. J. Kubber, ${ }^{2}$ G. Han, ${ }^{3}$ S. Rigamonti, ${ }^{4}$ D. Sanchez-Portal, ${ }^{4}$ D. Enders, ${ }^{3}$ \\ A. Pucci, ${ }^{1}$ and T. Nagao ${ }^{3, a)}$ \\ ${ }^{1}$ Kirchhoff Institute of Physics, University of Heidelberg, Im Neuenheimer Feld 227, \\ D-69120 Heidelberg, Germany \\ ${ }^{2}$ Department of Materials Science and Engineering, Massachusetts Institute of Technology, Cambridge, \\ Massachusetts 02139, USA \\ ${ }^{3}$ Center for Materials Nanoarchitechtonics, National Institute for Materials Science, 1-1 Namiki, Tsukuba, \\ Ibaraki 305-0044, Japan \\ ${ }^{4}$ Donostia International Physics Center (DIPC) and Centro de Física de Materiales CSIC-UPV/EHU, \\ Materials Physics Center (MPC), Paseo Manuel de Lardizabal 4-5, San Sebastián E-20018, Spain
}

(Received 16 January 2010; accepted 21 May 2010; published online 14 June 2010)

\begin{abstract}
Infrared spectroscopy is demonstrated to sensitively detect electronic excitations in 1-nm-wide wires made of indium. The polarization-dependent spectra measured at room temperature show a strong broadband plasmonic absorption feature in the direction parallel to the wires, while in the perpendicular direction the wires stay nearly transparent in the same spectral range. At $88 \mathrm{~K}$ the wires do not show this broadband absorption anymore, but instead, several interband-transition features arise for both polarizations, in agreement to the gap opening of the metal-to-insulator transition as known for this one-dimensional structure. (C) 2010 American Institute of Physics. [doi:10.1063/1.3451461]
\end{abstract}

Infrared (IR) spectroscopy is a sensitive tool for materials research down to the nanometer scale. Besides its high spectral resolution, one of the biggest advantages is its accessibility to low energy electronic excitations and to the metallic conductivity, ${ }^{1,2}$ which is of special interest for characterizing future nanoscale device materials.

In the present letter, we report on the IR spectroscopic detection of electronic excitations in 1-nm-wide In atomic wires on a $\mathrm{Si}$ substrate. At room temperature (RT), we observed a strong and broad absorption feature for the electric field along the wire direction, but in the wire-perpendicular direction only marginal absorption was observed. For both polarization directions, the RT spectra do not show any prominent feature associated with interband transitions. However, upon cooling down to $88 \mathrm{~K}$, the strong and broad feature disappears and weaker but sharper absorption bands appeared at 0.48 and $0.72 \mathrm{eV}$, which are attributed to interband transitions of this system. ${ }^{3,4}$ The temperature dependence of the IR spectra is consistent with the excitation of one-dimensional plasmons in In wires (with finite length) at RT and their disappearance at low temperature (LT) due to the metal-to-insulator transition.

The experiments were conducted under ultrahigh vacuum conditions at a base pressure of $1 \times 10^{-10}$ mbar. The chamber is equipped with a liquid nitrogen cryostat and connected to a nitrogen purged Fourier-transform IR spectrometer (Nicolet-Japan, NEXUS670) via ZnSe windows. Surface morphology at RT and LT is investigated by reflection highenergy electron diffraction (RHEED). The periodic array of In-atom chains is self assembled onto a vicinal $\mathrm{Si}(111)$ wafer $\left(12 \times 5 \mathrm{~mm}^{2}\right)$, with miscut angle of $0.9^{\circ}$ toward the [112] direction. The $\mathrm{Si}$ wafer was cleaned by standard flash heating, ${ }^{5}$ and about 0.9 monolayers (ML) of In were depos-

${ }^{a)}$ Electronic mail: nagao.tadaaki@nims.go.jp. ited onto the cleaned substrate at $350{ }^{\circ} \mathrm{C}$. In was evaporated from an alumina coated tungsten basket at deposition rate of $0.2 \mathrm{ML} / \mathrm{minute}$. Due to the template effect of the vicinal wafer, In chains were aligned along the step direction and form a macroscopic single-domain surface superstructure with $4 \times 1$ periodicity. ${ }^{6,7}$ IR spectroscopy at RT and at $88 \mathrm{~K}$ was performed in transmittance geometry (at normal incidence, $8 \mathrm{~cm}^{-1}$ resolution) with a polarizer used for either parallel or perpendicular excitation regime of electric field with respect to the atom-chains. Since the In-nanostructure layer is much thinner than the IR wavelength, the decrease in transmittance due to the In structure is linearly proportional to its dynamic conductivity ${ }^{2}$ or, more simply spoken, to the absorption by the In chains. The IR beam with a diameter of $4 \mathrm{~mm}$ probes an area where the In structure is formed uniformly. Relative spectra were obtained by dividing the spectra of the In chains on $\mathrm{Si}$ by the spectrum of the clean $\operatorname{Si}(111)-7 \times 7$ (measured before In deposition) at the same temperature. The stability of the entire set up was checked prior to the spectral measurements. The baseline intensity drift with time is below $0.1 \%$ of transmittance per one hour.

The (relative) transmittance spectra at RT for different polarization reflect the anisotropy of the conductivity of the In wires. As can be seen in Fig. 1, the transmittance shows a strong absorption for electric field in the chain direction (parallel polarization, $\bar{\Gamma} \overline{\mathrm{X}}$ direction). On the contrary, for the perpendicular polarization direction ( $\overline{\mathrm{X}} \overline{\mathrm{M}}$ direction) the system stays almost transparent in the same frequency range. Because of the low energy and its strong anisotropy, the observed feature must be related to the one-dimensional metallic band of the In wires $3,7,8$ which is shown in the electronic band calculation [see Fig. 1(b)]. For polarization parallel to the chains, a Drude-type metallic tail from the dc to the visible range is expected. However, surprisingly, we observed a broad resonance-like absorption signal [Fig. 1(c): 
(a)

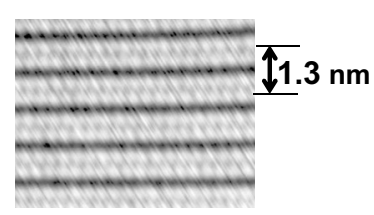

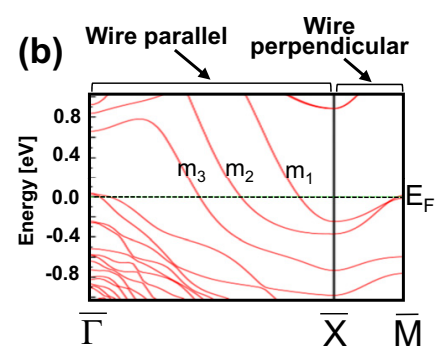

Energy [eV]

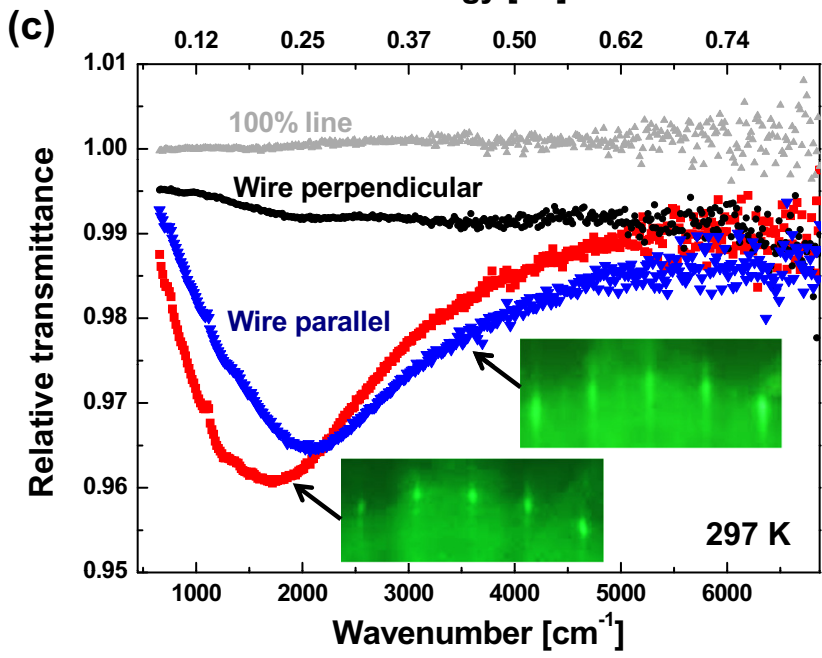

FIG. 1. (Color online) (a) A scanning tunneling microscope picture of In chains at RT $\left(6.5 \times 6.5 \mathrm{~nm}^{2}\right)$. (b) Calculated band structure along $(\bar{\Gamma} \overline{\mathrm{X}})$ and across $(\overline{\mathrm{X}} \overline{\mathrm{M}})$ the In chains. Along the $\bar{\Gamma} \overline{\mathrm{X}}$ direction the three electronic bands $\mathrm{m}_{1}, \mathrm{~m}_{2}$, and $\mathrm{m}_{3}$ cross the Fermi level, which produces metallic character. (c) IR transmittance of two differently prepared samples at RT with electrical field polarized parallel (red squares and blue triangles) and perpendicular (black circles) to the In-atom chains, respectively. The 100\% line (gray dots) reveals the stability of the system right before the experiments.

red squares] centered at $0.2 \mathrm{eV}$ and vanishing absorption toward lower wave numbers. The transparent behavior at low wave numbers and the $0.2 \mathrm{eV}$ absorption feature might be attributed to the existence of a $0.2 \mathrm{eV}$ band gap at the Fermi level. However, such assignment contradicts many preceding experiments that showed nonvanishing density of states at the Fermi level of the RT phase. ${ }^{6}$ Additionally, the observed RT feature is too broad and too strong compared to typical interband transitions.

It is more likely that the observed feature belongs to a low-energy plasmonic excitation in atom wires. ${ }^{7,9}$ For the plasmon wavelength longer than a characteristic chain length $L$, the plasmon wavelength stays at the value for $q=\pi / L$, where a standing wave is formed, which allows IR optical detection. For example, for finite-size $\mathrm{Ag}$ particles it was experimentally shown that the plasmonic frequency below $q<\pi / L$ stays at the value for $q=\pi / L .^{10}$ For the In-atom chains, the dispersion curve from electron energy loss spectroscopy (EELS) (Ref. 7) extends to a lowest plasmon energy (that could be detected separately from elastic reflection) of about $150 \mathrm{meV}$ for a momentum of $q=0.0033 \AA^{-1}$. According to $q=\pi / L$, the chains are at least about $100 \mathrm{~nm}$ long in that EELS study. One has to take into account that this estimate of $L$ is a rather rough one since the plasmon dispersion curve in Ref. 7 is very steep and the experimental energy resolution was limited. According to $q=\pi / L$ and referring to the dispersion relation of Ref. 7, it follows an average-length
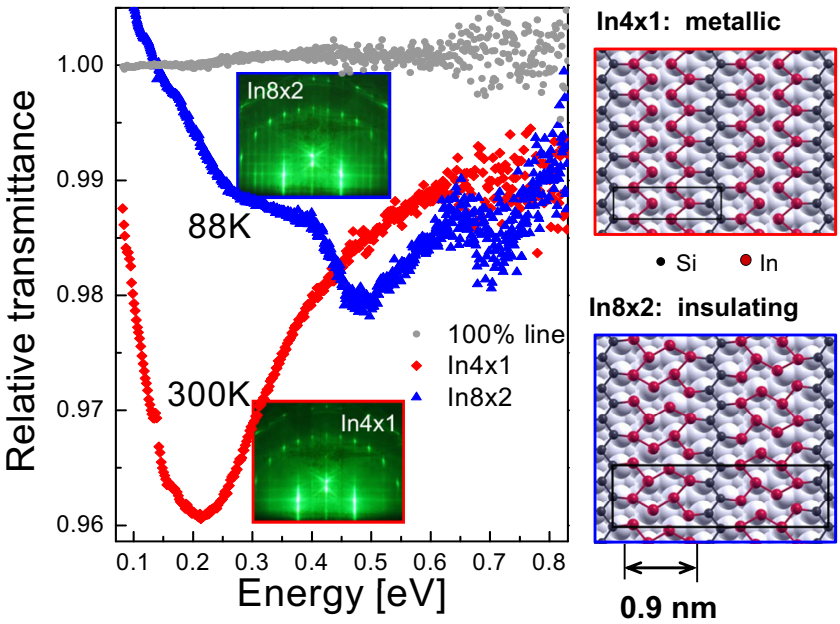

FIG. 2. (Color online) (Left) The metal to insulator transition of In wires on $\mathrm{Si}$ (the transition from $4 \times 1$ at RT to $8 \times 2$ at LT) shown by the two different IR transmittance spectra (both for polarization parallel to chains) together with the RHEED patterns. (Right) Optimized structures for the two phases as obtained in our calculations. Unit cells are indicated by solid lines. The structural transition proceeds via the dimerization of the outer In rows together with a shear distortion of neighboring $4 \times 1$ zigzag chains (Ref. 3 ).

estimate of about $L=55 \mathrm{~nm}$ from the resonance in our IR spectrum at RT, which is in reasonable accordance with the $L$ estimate from EELS data. The expected relationship between In-chain length $L$ and IR resonance gets further support from IR spectral observations on smaller $4 \times 1$ superstructures of samples prepared with annealing temperatures below $350{ }^{\circ} \mathrm{C}$, see blue triangles in Fig. 1(c). The RHEED spots from these samples are broader than the former ones and the IR resonance appeared at about $0.25 \mathrm{eV}$, from which we can roughly estimate a mean $L$ of about $48 \mathrm{~nm}$. Our findings on finite metallic chains are in general accordance with theoretical studies of fundamental plasmon resonances in Na-atom chains ${ }^{11}$ that show a blueshift in the longitudinal mode (polarization parallel to the chain) with decreasing $L$. For polarization perpendicular to the chain, plasmonic excitation (the transverse plasmon mode) is at much higher energies for the same $L$ and has much lower oscillator strength. ${ }^{11}$ Thus, the transparency of the In chains on silicon at RT for polarization perpendicular to the chains is in agreement to the calculated $\mathrm{Na}$-chain behavior. The plasma frequency (parameter of the Drude model ${ }^{2}$ ) for the In chains as derived from the oscillator strength associated with electron or hole pockets ${ }^{12}$ in chain direction is $\omega_{\mathrm{P} \|}=48420 \mathrm{~cm}^{-1}$. This value is comparable to typical metal data and thus allows us to compare the present system to other metal atom chains like those of $\mathrm{Na}$ atoms.

Figure 2 compares spectra of the In chains for different temperatures under electric field parallel to the chains. The strong spectral changes are attributed to the phase transition from In $4 \times 1(\mathrm{RT})$ to In $8 \times 2(\mathrm{LT}, 88 \mathrm{~K})$. This metal-toinsulator transition is associated with a periodic lattice distortion, as indicated in Fig. 2. The In $8 \times 2$ phase does not show anymore the strong and broad plasmonic feature around $0.2 \mathrm{eV}$ but a weaker and broader one at about $0.26 \mathrm{eV}$ and two very significant absorption peaks at 0.48 and $0.72 \mathrm{eV}$.

Figure 3 shows the LT IR spectra of the In $8 \times 2$ phase for the two different polarization directions. The inset (upper right) provides the calculated band structure using the SIESTA 


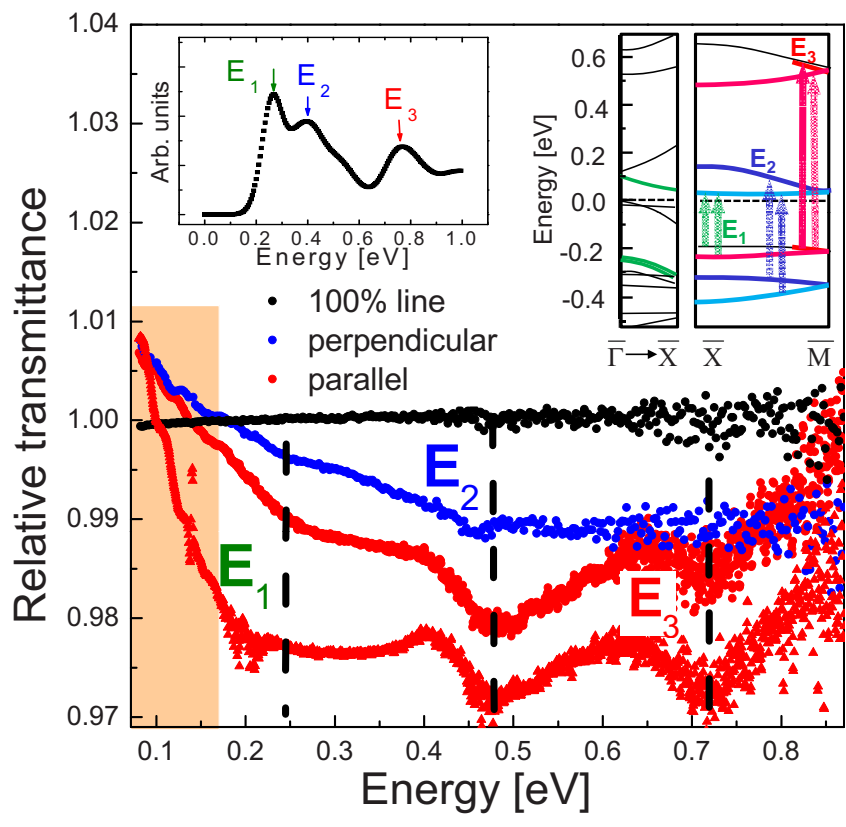

FIG. 3. (Color online) LT relative IR transmittance of the $8 \times 2$ phase for parallel (red circles and triangles) and perpendicular (blue) polarization. For parallel direction, spectra from two different samples are shown. The insets show the calculated band structure of the $8 \times 2$ phase near $\bar{\Gamma}$ and along $\bar{X} \bar{M}$ with the assignments of the different transitions as obtained from our theoretical analysis, and the calculated optical conductivity spectrum (with Gaussian broadening of $50 \mathrm{meV}$ ).

code $^{13}$ for the In chains on a five-bilayers $\mathrm{Si}$ slab with a $\mathrm{k}$-sampling equivalent to $256 \mathrm{k}$-points in the $(1 \times 1)$ unit cell. The large number of k-points and relatively thick slabs are crucial for convergence of the results, furthermore, to obtain a fully converged excitation spectrum we have used up to 1444 k-points in the $8 \times 2$ Brillouin-zone (inset, upper left). In the IR spectra for the two polarization directions we see similar spectral structures with a stronger absorption for polarization along the chain direction. Below $0.15 \mathrm{eV}$, the In $8 \times 2$ structure for both the polarization directions is more transparent than the $7 \times 7 \mathrm{Si}$ surface, which indicates the insulating character of the $8 \times 2$ surface, since the $7 \times 7 \mathrm{Si}$ has metallic surface states. In Fig. 3, the observed transitions are assigned to interband transitions from the nearly parallel bands close to the Fermi level and nearby the $\bar{X} \bar{M}$ direction (see inset). This identification is based both on the values of the energies and on a detailed analysis of the values of dipolar transition matrix elements. Indeed, the calculated dynamic conductivity for the $8 \times 2$ structure (see inset) is in rather good agreement with the experimental results. It exhibits three clear peaks at $0.28,0.40$, and $0.77 \mathrm{eV}$, that can be identified with the $\mathrm{E}_{1}, \mathrm{E}_{2}$, and $\mathrm{E}_{3}$ experimental transitions, and a negligible absorption below $0.15 \mathrm{eV}$. In the experiment, the $\mathrm{E}_{1}$ peak is somewhat broader and less intense than the calculation. This can be due to both, the limitations of density functional calculations and the observed preparation dependence of $E_{1}$.
In the experimental spectra, the absorption structure at $0.26 \mathrm{eV}\left(\mathrm{E}_{1}\right)$ is rather broad. We found typical values for the maximum of $\mathrm{E}_{1}$ between 0.2 and $0.3 \mathrm{eV}$. The position of this absorption maximum depends on preparation details that can introduce imperfections or the coexistence of different structural phases like $\sqrt{ } 31 \times \sqrt{ } 31$ and differently oriented $4 \times 1$ domains. Impurity and imperfection related issue was reported previously ${ }^{14}$ and the calculated band structure indeed shows a strong dependence on the smallest structural details. Furthermore, the imperfections can make some transitions visible that were optically silent originally. In particular, the transitions around $\bar{\Gamma}$, although slightly larger in energy and with negligible spectral weight for the pristine surface, could contribute with a broad feature near $0.3 \mathrm{eV}$ because of the larger dispersion compared to the bands nearby $\overline{\mathrm{X}} \overline{\mathrm{M}}$.

In summary, IR spectroscopy was demonstrated to be a powerful tool to detect the low-energy electronic features of 1-nm-wide metallic wires on IR-transparent $\mathrm{Si}$. In the RT metallic phase, In wires produce strong IR absorption with high anisotropy; an unexpected resonance-like feature appears that we assigned to plasmonic excitations in finitelength atom chains. In the LT phase, sharp absorption features were observed at 0.48 and at $0.72 \mathrm{eV}$ and a structuresensitive one at about $0.26 \mathrm{eV}$, in quite reasonable agreement with the theoretical predictions. These features show little anisotropy and are attributed to interband transitions.

This work is a part of the joint project "Strategic International Cooperative Program" funded by DFG-Germany (Grant No. PU193/9) and JST-Japan, and Spanish MICINN (Grant No. FIS2007-66711-C02-02) and Grant-in-Aid for Scientific Research from the Ministry of Education, Culture, Sports, Science, and Technology (MEXT).

${ }^{1}$ M. Klevenz, F. Neubrech, R. Lovrincic, A. Pucci, and M. Jalochowski, Appl. Phys. Lett. 92, 133116 (2008).

${ }^{2}$ R. Lovrinčić and A. Pucci, Phys. Rev. B 80, 205404 (2009).

${ }^{3}$ C. González, J. Guo, J. Ortega, F. Flores, and H. H. Weitering, Phys. Rev. Lett. 102, 115501 (2009).

${ }^{4}$ S. Chandola, K. Hinrichs, M. Gensch, N. Esser, S. Wippermann, W. G. Schmidt, F. Bechstedt, K. Fleischer, and J. F. McGilp, Phys. Rev. Lett. 102, 226805 (2009).

${ }^{5}$ J. Viernow, J.-L. Lin, D. Y. Petrovykh, F. M. Leibsle, F. K. Men, and F. J. Himpsel, Appl. Phys. Lett. 72, 948 (1998).

${ }^{6}$ H. W. Yeom, S. Takeda, E. Rotenberg, I. Matsuda, K. Horikoshi, J. Schaefer, C. M. Lee, S. D. Kevan, T. Ohta, T. Nagao, and S. Hasegawa, Phys. Rev. Lett. 82, 4898 (1999).

${ }^{7}$ C. Liu, T. Inaoka, S. Yaginuma, T. Nakayama, M. Aono, and T. Nagao, Phys. Rev. B 77, 205415 (2008).

${ }^{8}$ J. R. Ahn, J. H. Byun, H. Koh, E. Rotenberg, S. D. Kevan, and H. W. Yeom, Phys. Rev. Lett. 93, 106401 (2004).

${ }^{9}$ T. Nagao, S. Yaginuma, T. Inaoka, and T. Sakurai, Phys. Rev. Lett. 97, 116802 (2006).

${ }^{10}$ F. Moresco, M. Rocca, T. Hildebrandt, and M. Henzler, Phys. Rev. Lett. 83, 2238 (1999).

${ }^{11}$ J. Yan, Z. Yuan, and S. Gao, Phys. Rev. Lett. 98, 216602 (2007).

${ }^{12}$ X. Lopez-Lozano, A. A. Stekolnikov, J. Furthmuller, and F. Bechstedt, Surf. Sci. 589, 77 (2005)

${ }^{13}$ J. M. Soler, E. Artacho, J. D. Gale, A. García, J. Junquera, P. Ordejón, and D. Sánchez-Portal, J. Phys.: Condens. Matter 14, 2745 (2002).

${ }^{14}$ T. Tanikawa, I. Matsuda, T. Kanazawa, and S. Hasegawa, Phys. Rev. Lett. 93, 016801 (2004). 